\title{
An open risk index with learning indicators from OSM-tags, developed by machine learning and trained with the WorldRiskIndex
}

\author{
D. Feldmeyer ${ }^{1}$, H. Sauter ${ }^{1}$, J. Birkmann ${ }^{1}$ \\ ${ }^{1}$ IREUS, Institute of Spatial and Regional Planning, University of Stuttgart, Germany - Daniel.feldmeyer@ireus.uni-stuttgart.de
}

\author{
Commission IV, WG IV/4
}

KEY WORDS: WorldRiskIndex, Vulnerability, Risk Management, Machine Learning, OSM, R, QGIS, PostGIS

\begin{abstract}
:
While climate change is already a real issue in many parts of the world, it is even more threatening the well-being of future generations. The SDG 1.5 explicitly aims to reduce the vulnerability and exposure to climate related hazards by 2030. The World Risk Index (WRI) is one well-respected approach in profiling countries risk to natural hazard. To effectively monitor development and detect decision points on the climate resilience pathway, data of high resolution in space and time about the world's countries is of urgent importance. The World Risk Index will guide the supervised learning part resulting in an indicator set derived from OpenStreetMap (OSM) tags, establishing on one hand an open risk index and adding deep explanatory power to its components by a qualitative discussion of the OSM themes. The second part explores with unsupervised algorithms the inherent characteristic of country groups classified by the open risk index and deduces common patterns of socio-economic vulnerability. Hence, the inherent challenge of this work is to substitute existing static indicators with new dynamic indicators, not only substituting them but also painting a more detailed picture. Moreover, new data sources still questioned often by their reliability compared to World Bank or census data, and therefore its opportunities are neglected instead of critically exploring the potential. This unique combination is not done yet and bares huge potential moreover united with the open source geo community to contribute a little piece of the puzzle for achieving the SDG 1.5.
\end{abstract}

\section{INTRODUCTION}

Climate change is threatening human well-being in many parts of the world, as it often results in an interaction of natural hazards with the vulnerability of the society leading to disastrous impacts. While understanding the meteorological processes in the light of the unstationarity of climate change and its changing patterns, knowledge on the vulnerability as result of resilience and exposure becomes crucial in the process of adaptation (Birkmann 2006). Billions spent across Europe for structural protection measures have shown to result in higher monetary losses, partially explained with increased values exposed (Fuchs et al. 2017). Showing the need to for better informed decisions. Indicators are one way to assess, evaluate and monitor vulnerability and risk (Mach et al. 2016, Queiroz de Almeida et al. 2016). The WorldRiskIndex developed by Birkmann and Welle in cooperation with Alliance Development Works (see Birkmann et al. 2011) is one approach of operationalization of the risk formula (IPCC 2012) on country level for 171 countries. The index is based on 28 indicators from different global open data sources. The framework captures several facets of risk and the analysis allows to deduce profiles for countries showing problems in their coping capacity and susceptibility (Birkmann et al. 2011, Welle \& Birkmann 2015 a/b, Birkmann \& Welle 2016, Feldmeyer, Birkmann \& Welle 2018). This understanding of risk and vulnerability helps making better informed decisions; by selecting the right adaptation measures and monitoring its progress. The analysis of the World Risk data from 2012 - 2017 shows that despite a slide decrease in risk, the continental region of Oceania falls behind. Moreover, a high persistence of vulnerability in many African countries and its slow improvement, suggests that third-world countries are likely not able to implement integrated risk management strategies fast enough in the face of climate change (Feldmeyer, Birkmann \& Welle 2018). Many more approaches exist for assessing vulnerability showing the complexity of the phenomenon (Cutter et al. 2003, Birkmann 2006, Karagiorgos et al. 2016, Jamshed et al. 2017, Sorg et al. 2018). Despite the century of information, a lack of data is existing for measuring social capacities (Sorg et al. 2017). The social and crowd sourced OpenStreetMap database shows a huge potential for adding social aspects to the official governmental statistics with high spatial resolution. However, to the authors knowledge, a vulnerability index based on OSM has not yet been developed. Hence, this paper is exploring the potential of this unique geodatabase to assess and evaluate global vulnerability.

Consequently, the following objectives are targeted:

- Developing a methodology for data handling of the global OSM dataset and deducing country statistics.

- Developing a data driven machine learning methodology to model countries vulnerability to natural hazards.

- What are the main tags (key \& value) and keys explaining vulnerability on a country scale?

\section{METHODOLOGY}

\subsection{OSM data}

For this study, we count the numbers of unique tags from OSM nodes per country and statistically compare the counts with vulnerability indicators used in the World Risk Index. Although the spatial coverage and information density of OSM data varies across the world in relation to the number and performance of the national and regional contributors, its big advantage remains in the accessibility of a standardized global dataset.

The conceptual data model of OSM is built by elements that can be either nodes, ways or relations. Nodes, as the simplest form, representing only a point defined by coordinate pairs, can be connected to ways, which represent polylines (e.g. streets) or polygons (e.g. boundaries) if the lines are closed (endpoint=start 
point). Relations are multi-purpose data structures that define logical relationships between two or more elements. All of the aforementioned elements can be attributed with tags, i.e. plain text key-value pairs (e.g. amenity=restaurant) which further describe the meaning or function of objects. Whilst keys are unique and often categorial (landuse $=$, amenity $=, \ldots$ ), values can contain anything from individual names (e.g. name $=$ “Universität Stuttgart”, phone=+49 45627 2983) to numbers.

\subsection{Data preprocessing}

The OpenStreetMap dataset was downloaded as full planet file from https://planet.openstreetmap.org/ (date: 2019-04-25 00:58 UTC18:17). Due to the amount of information stored in the full OSM database (keys: 74.531, tags: 102.003.489; taginfo.openstreetmap.org - 2019-05-24 00:58 UTC, Data (C) OSM contributors (ODbL)), data reduction to a meaningful number of keys and values was crucial for this study. For this purpose the planetfile was processed with osmctools, a collection of command line programs and then imported into a PostgreSQL/POSTGIS database before the statistical analyses could be conducted. As a part of this preprocessing steps the osmfile was split into 197 single country files based on Natural Earth country polygons (NE_souvereignty - link). The aggregation to a summary table with tag counts per country was conducted in the PostgreSQL database. Figure 1 depicts the full preprocessing workflow.

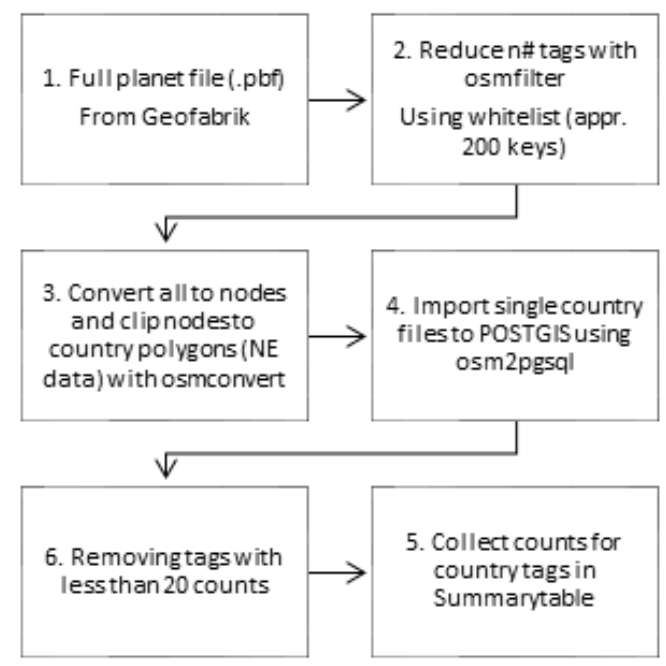

Figure 1: Data preprocessing workflow

In order to make as less assumptions in the first steps "all" remaining tags were treated as possible indicators to ensure the best possible exploration of the wealth of information by the OSM database and to detect potential new relations. Resulting in indicators of 190 by 250 nodes of tag counts.

The statistic were done in RStudio with $\mathrm{R}$ and several extensions (RStudio Team 2018, R Core Team 2019). Connecting to the database with the RPostgres package (Wickham, Ooms \& Müller 2018). To reduce the high dimensionality of the data, three steps were taken: Indicators with only low coverage of the world were removed. The threshold was set to $>50 \%$ coverage. Indicators with zero and near zero variance were also excluded. At last, the pair-wise correlation was calculated and in case of $>0.7$ removed (Kuhn et al 2017).

\subsection{Statistical Learning}

In general, statistical learning is divided into supervised learning and unsupervised learning. Supervised in the sense that the response is known, and we want to predict the response or understand the relationship of the predictors (inference) (James et al. 2013).

2.3.1 Supervised learning: Roughly speaking two categories of supervised learning methods exist, linear and non-linear methods. Linear models often are more robust and also a higher predictive accuracy than non-linear models like e.g. trees. But, linear models assume a linear relation and additivity. The Generalized Additive Models (GAM) are somehow in between. Allowing non-linearity but still assuming additivity. For exploring the data in face of unknown relation the "Lasso Regression" for linear and "Random Forest” for moving beyond linearity were selected.

2.3.2 Lasso Regression: Lasso regression is similar to ridge regression. Whereas in ridge regression all predictors are kept in the model and a shrinkage parameter determines their influence but never is Zero. Lasso regression uses a $l_{1}$ penalty forcing parameters to zero when the tuning parameter is large enough.

$\sum_{i=1}^{n}\left(y_{i}-\beta_{0}-\sum_{j=1}^{p} \beta_{j} x_{i j}\right)^{2}+\lambda \sum_{j=1}^{p}\left|\beta_{j}\right|=\operatorname{RSS}+\lambda \sum_{j=1}^{p}\left|\beta_{j}\right|$.

James et al. 2013

The "glmnet" package by Friedman, Hastie \& Tibshirani (2010) is used to model the lasso regression. The data is split into training and test data, by randomly selecting $50 \%$ of the rows as training data. The best lambda is chosen by 10 -fold cross validation.

2.3.3 Trees: Decision trees have some advantages and disadvantages over linear regression. First of all, decision trees are non-linear classifier in contrast to linear regression. Trees are understood very well also by non-statisticians as the graphical display is easy to understand. Simple trees can be sensitive to even small changes in data and have lower predictive accuracy. To overcome those shortcoming bagging, random forest and boosting of trees was developed and can substantially increase their performance (James et al. 2013).

As previous the data is split into training and test data. In a first exploratory step to get also a baseline and interpret the result of random forest a regression tree is calculated with the "tree" package (Ripley 2014). Secondly, for improving the result the regression tree is pruned. A very large tree is very likely to over fit the data and consequently a smaller tree could be favourable in means of variance at the cost of bias. One method is to grow a large tree and then to prune it back with the weakest link method.

Random Forest takes the advantages of the tree method and improves the weakness by building a forest. Each time when building a tree a bootstrapped training sample is taken. At each split also only a random sample of predictors is selected. This ensures that not one very strong predictor dominates all trees. The randomForest() function by Liaw and Wiener (2002) is on implementation of the method. 


\subsection{Unsupervised Learning}

Unsupervised learning summarizes method which do not predict a specific output, like vulnerability. They are method to reduce dimensions and uncover relations of the data. The question set up in this paper is, which factors of OSM are related to high vulnerable countries.

2.4.1 Principal Component Analysis: Principal Component Analysis (PCA) is beside Cluster Analysis one of the most common unsupervised methods, although can be also applied in a supervised way for e.g. regression.

Important for PCA is the data pre-processing as the scale influences the components and hence the data need to be normalized. The caret package provides the preProcess() function including the method "pca". The prcomb() function within the kazaam package is applied for the PCA (Schmidt et al 2017).

\subsection{World Risk Index}

The World Risk Index by Birkmann is one operationalization of the risk formula (Figure 2). On the left side the exposure component is built by five indicators describing human exposure to natural hazards (earthquake, cyclones, floods, droughts, sea level rise). It is the physical exposure of people per country on an annual average basis, reflecting the probability of the event (Welle \& Birkmann 2015).

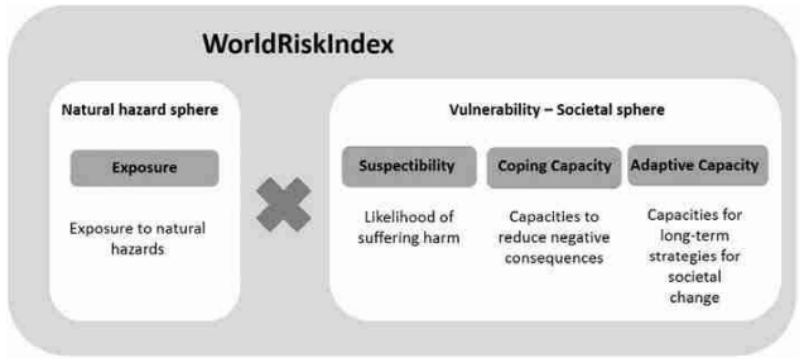

Figure 2. Risk formula of the World Risk Index (Welle \& Birkmann 2015b)

The socio-economic vulnerability (Table 1) consists of three pillars. First, Susceptibility, which describes the level to which people are affected by a hazard. Key components are Public Infrastructure, Housing conditions, Nutrition, Poverty and dependencies and the Economic capacity and income. Second, Coping capacity, how is the immediate ability to reduce the effects by the hazard. This strongly relies on Government and authorities, Disaster preparedness and early warning, Medical services, Social networks and Material Coverage. Third, Adaptive capacity, which resources has the society to plan and anticipate and reduce in the longer run negative impacts. Crucial within here are, Education, Gender equity, Environment, Adaptive strategies and investments.

The parts of the table with light grey underground are marked as parts which are not covered appropriate by official global data sources. The assumption made within this paper is that many of those components are being reflected in the OSM database in a direct and or indirect way. One method would have been to construct logically related indicators by thematic keys and tags.

Somehow rebuilt the concept of the World Risk Index indicator by indicator. This approach is limited in three ways. Firstly, the somehow chaotic contributions of slightly diverting spellings or reorder of key as a value or the other way around. Secondly, assumptions and judgements on the relations and their impact on vulnerability would introduce a strong bias. Thirdly, and most important the exploration of unknown and hidden patterns of the OSM spatial data is not obvious. Through the big-data characteristic of OSM data the uncovering needs advanced machine learning tools to extract unknown relationships and aspects of vulnerability. Therefore the combined vulnerability index was taken as the response variable and OSM-derived indicators as predictors.

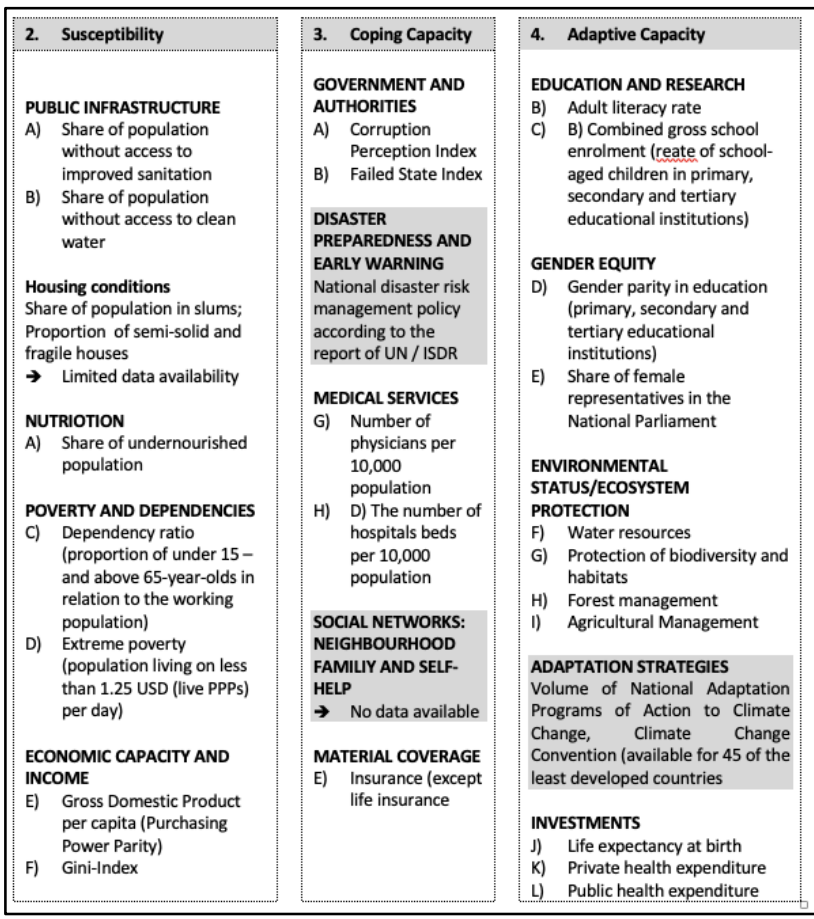

Table 1. Concept of the World Risk Index (Welle \& Birkmann 2015b)

\section{RESULTS}

The results are described in the logic given by the methodology. Starting with the dimensionality reduction, followed by the linear model of the lasso regression, moving beyond this linearity and concluding by detection of unsupervised patterns via PCA within the data.

\subsection{Dimension reduction}

Two datasets were derived, and results obtained based on them. First data set referred to as World Countries Tag Counts (Tags) with each country one row and 1341 columns representing each tag a column and the count of the tag per country as the value. As can be seen in the example of the 10 country and 3 columns shown in. Data set two is referred to as World Country Key Counts (Keys) the columns are merged per key and the counts of the value summed up for the count of the key. Which results in a much smaller dataset regarding columns of 110 but higher completeness.

After running the dimension reduction on the Tags, 99 tags as indicators remind. For the Keys, 27 variables remain and for Europe surprisingly only 35 variables are left compared to the 99 of the world. Finally, the counts per country were normalized by 
its population and min max normalization, so that all values are between 0 and 1 .

\subsection{Lasso regression}

The Lasso models linear combinations of the predictors on the response, in our case vulnerability. The lasso penalty "ell 1" shrinks the coefficients of some predictors exactly to zero reducing the predictors set and making the interpretation easier. The best Lambda is chosen by ten-fold cross validation of the training data.

The Mean-Squared-Error for the lambda selection cross validation process with the Keys data is forced to zero. As more and more predictors are forced to zero the error is reduced. Hence, it suggests that the relation of tag counts and vulnerability is not linear and a linear model therefore not appropriate for capturing it.

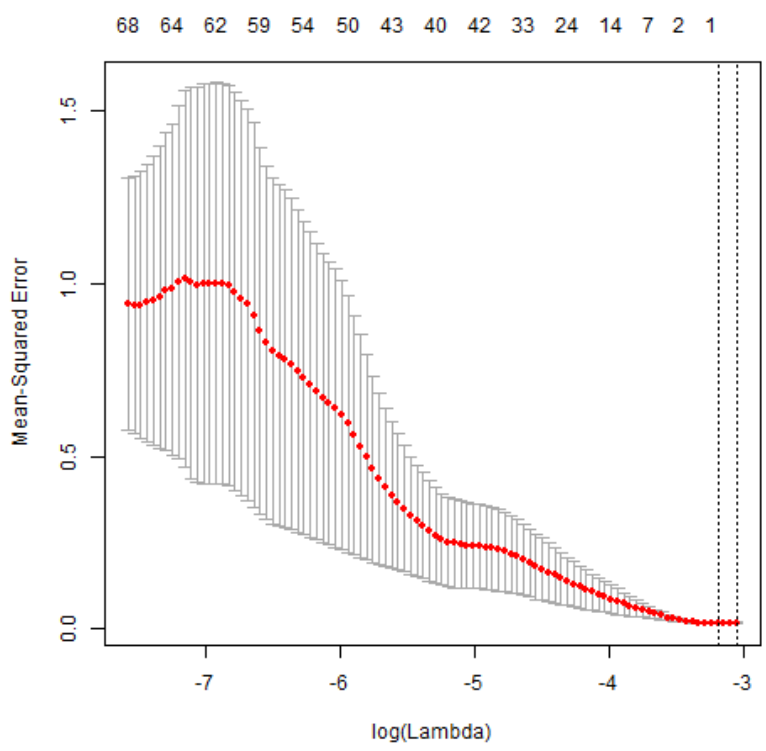

Figure 3. Lasso regression Lambda by crossvalidation Tags

Figure 3 shows the same Lambda selection for the Tags data. The Lambda is not pushed all to zero but nevertheless it suggests the pattern of the Keys, as it's the same data just split into the counts of key value pairs. In order to allow for non-linearity between counts per country and vulnerability a tree approach is chosen.

\subsection{Trees}

For all following trees as well as random forest, the columns of the two datasets served as predictors vulnerability of the World Risk Index as response variable.

3.3.1 Modelling vulnerability based on Key count per country: Three steps are run for each of the three datasets. First a simple regression tree is built. Second, this tree is pruned for better robustness and less variance. Third, a random forest is grown allowing multiple trees.

The performance of the trees on the Keys data. In a first simple step a regression tree was calculated (Figure 4). The top node divides the tree by traffic_signals into left and right. The right branch is further divided by the electric frequency of railways, buses, electric power supply networks and communication devices. Further distinguished with landuse, traffic_calming and operator.type. Where land use and traffic_calming are more or less self-explaining, the top linked values to the key operator:type are private, public, government and religious. On the left side again operator:type and traffic_calming appear.

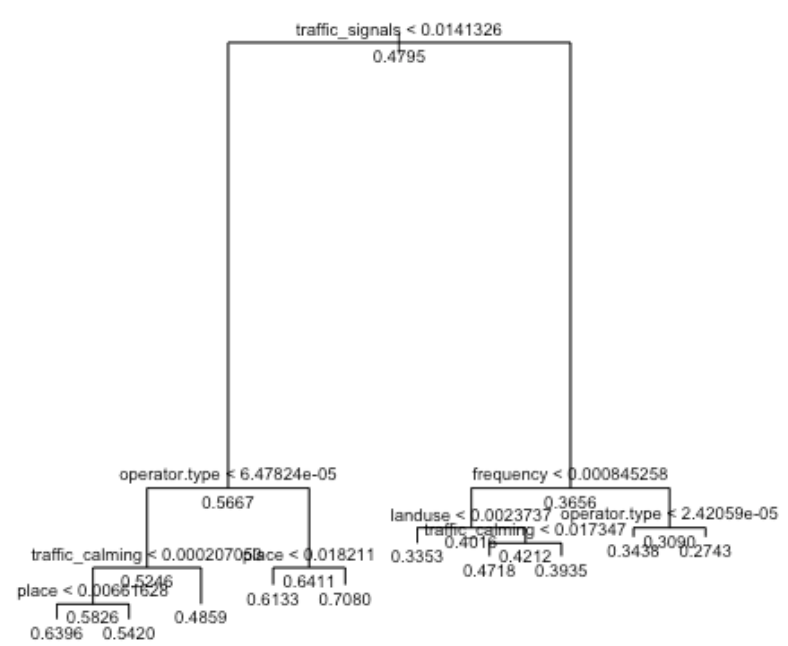

Figure 4. Regression Tree Keys

Pruning the regression tree to increase robustness and balance for the variance-bias trade-off is shown in Figure 5. This shows also the most relevant attributes for describing vulnerability with counts per key and country, which are traffic_signals, operator_type, traffic calming and frequency.

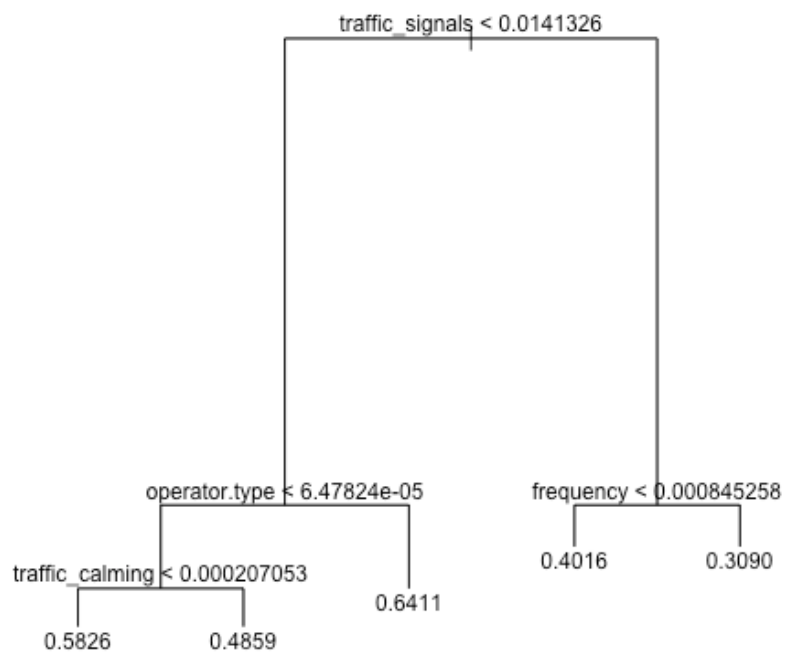

Figure 5. Pruned tree Keys

The 13 most important predictors for the random forest are shown in Table 2. The results come not as a surprise after the regression tree and the pruned tree. Nevertheless, the table gives a more detailed picture and in depth interpretation. The X.IncMSE is the increase in the MSE of the prediction, estimated by the out of bag cross validation. Again traffic_signals is the most important. Followed by traffic_calming, operator.type and landuse. Frequency appears slightly lower compared to the pruned tree.

Interesting is the last key with fire_hydrant.dyameter in the light of natural hazard and vulnerability. Such data are not in official country records. 


\begin{tabular}{l|ll}
\multicolumn{1}{c}{ X.IncMSE } & IncNodePurity \\
\hline traffic_signals & 20,0395575 & 0,430109572 \\
traffic_calming & 12,887251 & 0,265499982 \\
operator.type & 10,4132019 & 0,064276153 \\
landuse & 8,45125305 & 0,102887023 \\
service & 7,34750772 & 0,126863252 \\
designation & 7,09257133 & 0,084712005 \\
construction & 6,09648063 & 0,062480717 \\
frequency & 5,80008566 & 0,035168397 \\
vehicle & 5,0673767 & 0,04203698 \\
toll & 4,31681353 & 0,011428788 \\
smoothness & 4,20197549 & 0,020569056 \\
cables & 3,93164146 & 0,009381577 \\
fire_hydrant.diamet & 3,57858402 & 0,010674378 \\
er & &
\end{tabular}

Table 2: Random forest important keys

The statistical model is now used to predict vulnerability values of the world (Figure 6). As in the World Risk Index the quantile method is used for categorizing the countries. This of cource includes now test and training data. For the prediction accuracy error on the test data needs to be considered. Another additional model check is now the correct classified countries based on the model vulnerability scores classified by the quantile method. For the 169 countries $66 \%$ are ranked according to their World Risk Index classification.

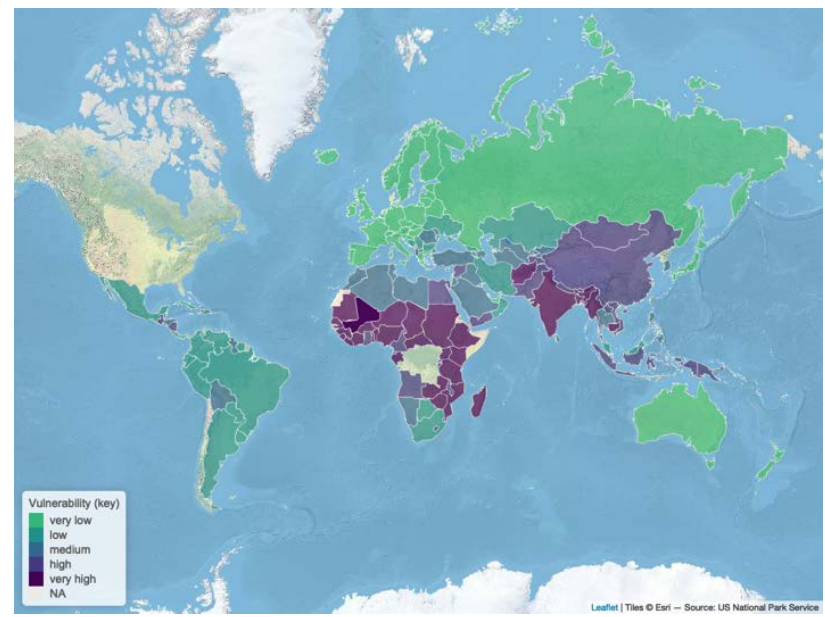

Figure 6. Map random forest model based on keys

3.3.2 Modelling vulnerability based on tag count per country: The database for the previous results were counts per key and country, which is a simple dimension reduction, additional to statistical dimension reduction. The following section now explores counts per tag as predictors of vulnerability.

In Figure 7 the regression tree of the Tags is split by shop_yes. The right brunch is further distinguished by shelter_type_public_transport. Further tags on this side are highway_rest_area, cuisine_mexican,shop_lottery and natural_volcano. The left side is contructed by the tags amenity_swimming_pool and highway_residential.

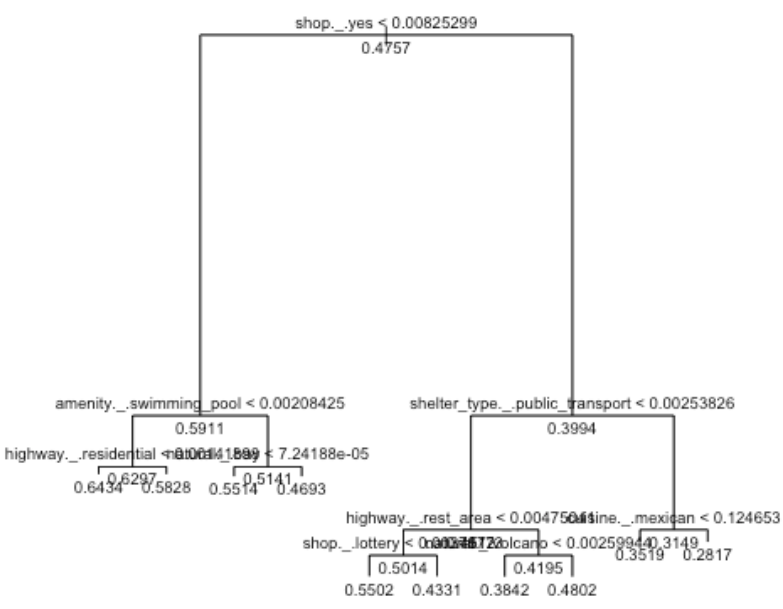

Figure 7. Regression Tree Tags

Pruning the tree as previous results in a simpler model (Figure 8). Hence the dominant tags for vulnerability are: shop_yes, amenity_swimming_pool, shelter_type_public_transport and highway_rest_area.

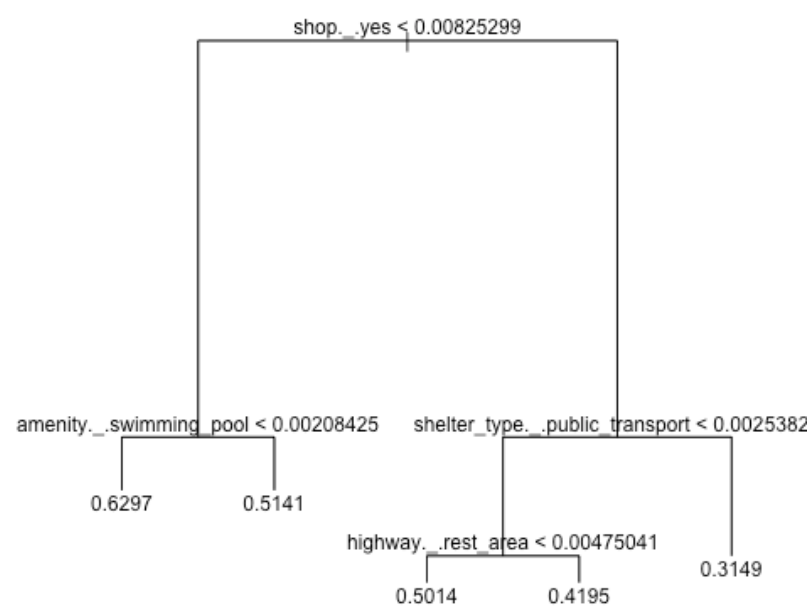

Figure 8. Regression Tree Tags

The random forest model goes hand in hand with the first two and fourth tag. Third tag although is amenity_shower. The tag swimming_pool appears much lower (Table 3).

IncMSE IncNodePurity

\begin{tabular}{l|ll} 
shop._.yes & 15,20 & 0,24 \\
leisure._.swimming_pool & 10,09 & 0,16 \\
amenity._shower & 9,07 & 0,15 \\
shelter_type._public_transpor & 8,58 & 0,09 \\
$t$ & 7,95 & 0,08 \\
sport._.basketball & 7,57 & 0,06 \\
man_made._.survey_point & 6,48 & 0,07 \\
cuisine._.mexican & 6,46 & 0,02 \\
cuisine._thai & 6,38 & 0,02 \\
barrier._.stile & 6,30 & 0,05 \\
landuse._.cemetery &
\end{tabular}




\begin{tabular}{l|cc} 
amenity._bureau_de_change & 5,94 & 0,03 \\
amenity._.swimming_pool & 5,83 & 0,02 \\
natural._.bay & 5,79 & 0,04
\end{tabular}

Table 3: Random forest important tags

Based on the random forest model the world vulnerabilities are calculated and mapped in Figure 9

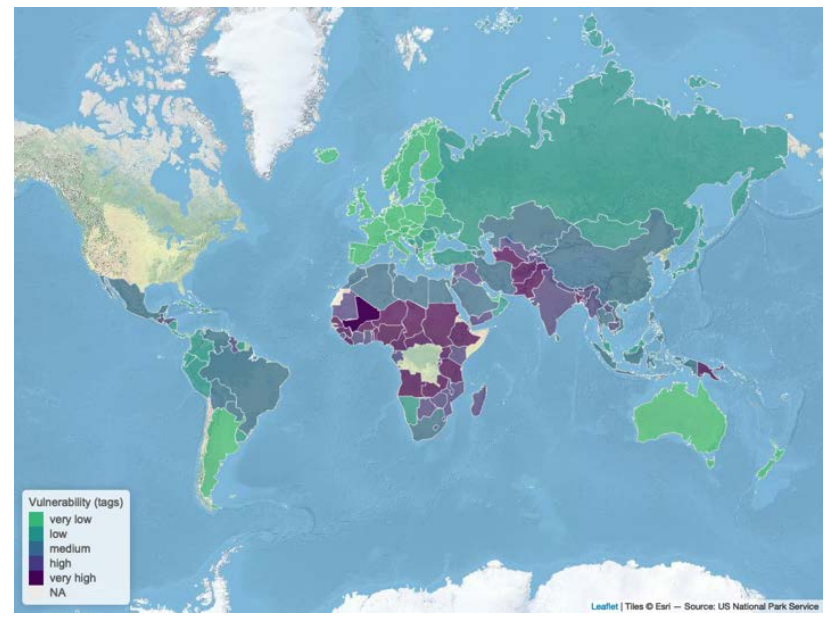

Figure 9. Map random forest model based on tags

Compared to the World Risk Index $65 \%$ of the countries are in the same category.

\subsection{Principal Component Analysis (PCA)}

Unsupervised learning explores underlying structures and combines sub-sets into new reduced dimensions. The 20 most vulnerable countries are selected and explored with the unreduced key counts and tag counts, 110 indicators respectively 1341 indicators. Afterwards zero and near zero variance predictors removed.

3.4.1 PCA Keys: The PCA is in the caret package part of the pre-processing function (Kuhn et al 2017). The function is set to explain $70 \%$ of the variance within the data.

The PCA for the keys resulted in seven components (Table 4). PC1 is highest loaded by smoking. sport and parking. PC2 is loaded by bridge, park_ride and smoothness. PC3 is loaded by junction, supervised and direction. The two highest predictors for PC4 are shelter and shelter_type. For PC5 the 2 highest are railway and aeroway. PC6 is dominated by genus, hiking and cuisine. Last, PC7 the two main predictors are horse and motorcycle.

\begin{tabular}{|c|c|c|}
\hline & Predictors & Name \\
\hline PC1 & $\begin{array}{l}\text { Smoking(no). sport and } \\
\text { parking }\end{array}$ & Health \\
\hline PC2 & $\begin{array}{l}\text { bridge, park_ride and } \\
\text { smoothness }\end{array}$ & $\begin{array}{l}\text { Infrastructure } \\
\text { Quality }\end{array}$ \\
\hline PC3 & $\begin{array}{l}\text { junction, supervised and } \\
\text { direction }\end{array}$ & Traffic Control \\
\hline PC4 & shelter and shelter_type & Shelter \\
\hline PC5 & railway and aeroway & Air \& Rail \\
\hline PC6 & genus, hiking and cuisine & Leisure \\
\hline PC7 & horse and motorcycle & Fun \\
\hline
\end{tabular}

3.4.2 PCA Tags: Removing from the tags the zero and near zero variance predictors 759 predictors remained. The loading of the single indicator is less strong than previous (Table 5). So more predictors contributing to each principle component. PC1: shop _ beverages, amenity _ arts_centre, leisure _ pitch, shop _ hardware, shop_doityourself, amenity _ kindergarten amenity_social_centre, information _ board, sport_soccer highway _ emergency_access_point - which could be summarized as Social, Art \& Shopping. PC2, is influenced quite low equally by 130 predictors of Tourism \& Sport. PC3 is hard to interpret, but best summarized as Tourist Infrastructure. PC4 is Man-Made. PC5 is Cuisine. PC6 summed as Health \& Leisure. PC7 is again loaded by man Historic \& Natural.

\begin{tabular}{ll}
\multicolumn{2}{c}{ Name } \\
\cline { 1 - 1 } PC1 & Social, Art \& Shopping \\
\cline { 1 - 1 } PC2 & Tourism \& Sport \\
\cline { 1 - 1 }$P C 3$ & Tourist Infrastructure \\
\cline { 1 - 1 }$P C 4$ & Man-Made \\
\cline { 1 - 1 }$P C 5$ & Cuisine \\
\cline { 1 - 1 }$P C 6$ & Health \& Leisure \\
\cline { 1 - 1 }$P C 7$ & Historic \& Natural \\
\cline { 1 - 1 } & Table 5 Principal Components of tags
\end{tabular}

\subsection{Moving from vulnerability to risk}

Modelling the exposure component of the World Risk Index only $35 \%$ of the countries were correctly classified. It was expected that the probability of affected people is not dependent on structural elements. Hurricanes, floods, drought and sea level rise are not map elements. But shouldn't they be?

3.5.1 Mapping Open Risk and World Risk: To Map the open risk index, the exposure from the World Risk is taken and combined by multiplying it with the key-based random forest model representing vulnerability.

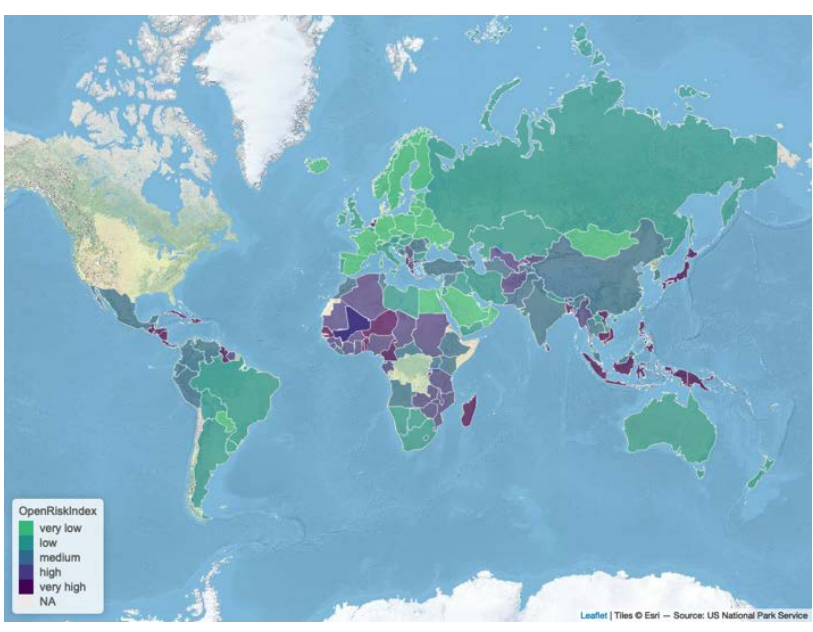

Figure 10. Open Risk Index

Figure 10 the world risk to natural hazard. The classification is adopted from the World Risk Index by the quantile method. 


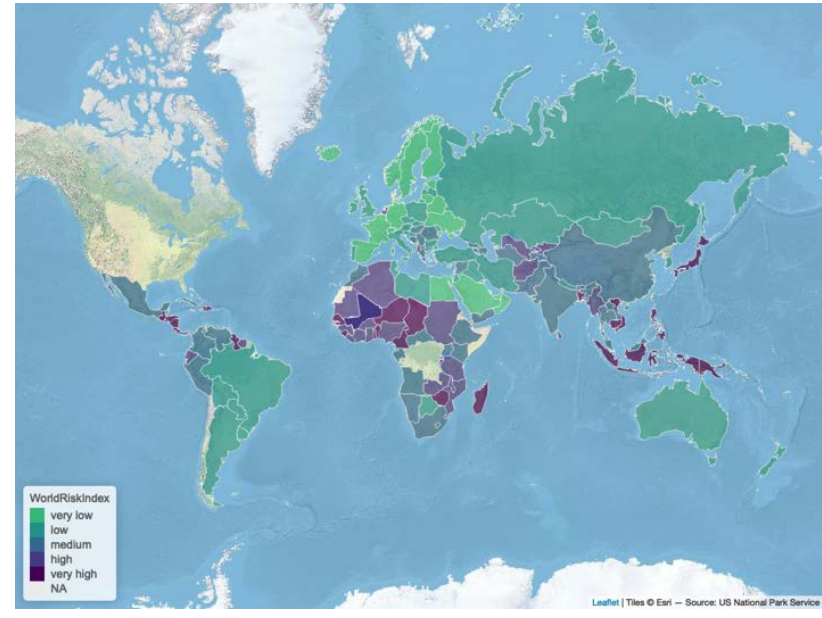

Figure 11. Vulnerability of the Open Risk Index

Figure 11 in comparison is the original World Risk Index. Obviously, the main patterns are coherent. Most of the differences are within the range of on class. Globally there is a persistent high risk in Oceania, Africa and Central America.

\section{DISCUSSION}

The approach provided meaningful insights into three questions: A) global country data deduced from OSM B) statistical learning for predicting vulnerability C) global vulnerability.

Firstly, the tag count per country seemed mostly influenced by the OSM coverage of this country. Population or absolute count could not remove this effect entirely. Additional spellings and translations could not be assessed during this study. These limitations need further consideration for global analysis. However, global datasets are sparse and the global coverage of the OSM data, combined with its availability represent a huge potential for risk management evaluation and global climate change adaptation studies.

Secondly, in this study the vulnerability index was considered as truth. However, uncertainties remain and further research on the propagation of these unknowns could clarify interpretations. As such, negative correlated indicators potentially exist and need to be further pointed out to improve the explanatory power of the indicators and by this of the risk index. Thus, there might remain hidden or obscured effects through the characteristic of the dataset and the current approach.

Thirdly, hazard could not be modelled. Therefore, the question remains if the information extracted from OSM does not yet include information which explicitly or implicitly is related to exposure or the methodology applied was not able to uncover those structures.

Fourthly, the WorldRiskIndex is on a continuous scale from zero to one. Another way instead could be also to model not the vulnerability index value but to classify instead or model its components separately and then also index them.

Fourthly, the preliminary results suggest that on a country scale its vulnerability can be approximated based OSM map elements and match key elements of the World Risk Index vulnerability (Table 1). Several infrastructure related tags (traffic_signals, traffic_calming, toll, smoothness) could be seen as approximation for the development of the country (ECONOMIC CAPACITY AND INCOME) but also the ability of the government to provide public goods (GOVERNMENT AND AUTHORITES, PUBLIC INFRASTRUCTURE). POVERTY AND DEPENDENCIES, MEDICAL SERVICES and HEALTH, are predicted by leisure, health and sport keys and tags (cuisine, leisure_swimming pool, sport_basketball). Disaster preparedness not covered in the World Risk Index due to lack of data might be approximated by shelter_public or fire_hydrant.diameter. Hence, not only vulnerability can be estimated but also the thematically important themes are covered by the prediction models.

Fifthly, the preliminary results of the PCA suggest that vulnerability can be explained by Health, Infrastructure Quality/Traffic Control and Shelter mainly. Argumentum e contrario considering the global effort in reducing vulnerability showing the importance of investments into health, infrastructure but also shelter for risk preparedness. More in general related to leisure and tourism the importance of a strong middle class.

Lastly, having said this, the focus of the study was to develop a methodology and find preliminary variables explaining vulnerability. In a second step beyond the scope of this paper now, the results need to be further tested.

\section{CONCLUSION}

The OpenRiskIndex is the first approach modelling socioeconomic vulnerability to natural hazards with statistical learning based on the OSM database.

Using counts per country as predictors is a possibility to deduce meaningful information without a super computer from the global OSM dataset. The price paid is of course the spatial explicitness (resolution). Supervised and unsupervised statistical learning proved to be able to model country socio-economic characteristics of vulnerability. Most significant dimensions were regarding health, infrastructure and economic capacity for explaining vulnerability. Although questions remain and could not be addressed in this study, OSM seems to offer the possibility of effectively monitoring changes in global vulnerability. Compared to country statistics and global databases where indicators often include a time lack and moreover are based on different years, covering or delaying the detection of trends, the very much alive OSM might be able to provide better coverage of a changing world.

Socio-economic indicators can provide valuable insides. Hence robust OSM based indicators for the SDG's, vulnerability and risk. Further research although is needed to develop a commonly accepted methodology and a global open source database with computational power to overcome the limitation of desktop pcs and fully explore the possibilities.

\section{REFERENCES}

Birkmann, J. 2006. Measuring vulnerability to natural hazards: towards disaster resilient societies. No. Sirsi) i9789280811353.

Birkmann, J., Welle, T., Krause, D., Wolfertz, J., Suarez, D., \& Setiadi, N., 2011. WorldRiskIndex: Concept and Results. In WorldRiskReport, pp. 13-43. Berlin: Alliance Development Works.

Birkmann, J., Cardona, O.D., Carreño, M.L., Barbat A.H., Pelling, M., Schneiderbauer, S., Kienberger, S., Keiler, M., Alexander, D., Zeil, P. and Welle, T., 2013: Framing vulnerability, risk and societal responses: The MOVE framework. Natural Hazards 01/2013; 67(2):193-211.

Birkmann J, Welle T., 2016. The World Risk Index 2016: reveals the necessity for regional cooperation in vulnerability reduction. J Extreme Events. https://doi.org/10.1142/S2345737616500056 
Fuchs S, Ro“thlisberger V, Thaler T, Zischg A, Keiler M (2017) Natural hazard management from a coevolutionary perspective: exposure and policy response in the European Alps. Ann Am As Geogr 107 (2):382-392.

https://doi.org/10.1080/24694452.2016.1235494

Cutter, S., Boruff, B., Shirley, W., 2003. Social Vulnerability to Environmental Hazards*. In: Social Science Q 84 (2), S. 242261. DOI: $10.1111 / 1540-6237.8402002$

Feldmeyer, D., Birkmann, J., Welle, T., 2017. Development of Human Vulnerability 2012-2017. In: Journal of Extreme Events 04 (04), S. 1850005. DOI: 10.1142/S2345737618500057.

IPCC [Intergovernmental Panel on Climate Change], 2012. Glossary of terms. In: Field CB, Barros V, Stocker TF, Qin D, Dokken DJ, Ebi KL, Mastrandrea MD, Mach KJ, Plattner G-K, Allen SK, Tignor M, Midgley PM (eds) Managing the risks of extreme events and disasters to advance climate change adaptation. A special report of working groups I and II of the Intergovernmental Panel on Climate Change (IPCC). Cambridge University Press, Cambridge, pp 555-564

James, G., Witten, D., Hastie, T., \& Tibshirani, R., 2013. An introduction to statistical learning . Corrected at 8th printing 2017. New York: springer.

Jamshed, A., Rana, I.A., Mirza, U.M., Birkmann, J., 2019. Assessing relationship between vulnerability and capacity: An empirical study on rural flooding in Pakistan. Int. J. Disaster Risk Reduct., 36.

Friedman, J., Hastie, T., Tibshirani, R., 2010. Regularization Paths for Generalized Linear Models via Coordinate Descent. Journal of Statistical Software, 33(1), 1-22. URL http://www.jstatsoft.org/v33/i01/.

Karagiorgos, K., Thaler, T., Heiser, M., Huebl, J., Fuchs, S., 2016. Integrated flash flood vulnerability assessment: insights from East Attica, Greece. J Hydrol 541(Part A):553-562. https://doi.org/10.1016/j.jhydrol. 2016.02.052

Kuhn, M., Contributions from Jed Wing, Steve Weston, Andre Williams, Chris Keefer, Allan Engelhardt, Tony Cooper, Zachary Mayer, Brenton Kenkel, the R Core Team, Michael Benesty, Reynald Lescarbeau, Andrew Ziem, Luca Scrucca, Yuan Tang, Can Candan and Tyler Hunt. (2017). caret: Classification and Regression Training. R package version 6.0-78. https://CRAN.Rproject.org/package $=$ caret

Liaw, A., Wiener, M., 2002. Classification and Regression by randomForest. R News 2(3), 18--22.

Mach, K., Mastrandrea, M., Bilir, T., \& Field, C., 2016. Understanding and responding to danger from climate change: the role of key risks in the IPCC AR5. Climatic Change, 136 (34), 427-444

Queiroz de Almeida, L., Welle, T. and Birkmann, J., 2016. Disaster risk indicators in Brazil: A proposal based on the world risk index. International Journal of Disaster Risk Reduction 17, 251-272

R Core Team, 2019. R: A language and environment for statistical computing. R Foundation for Statistical Computing, Vienna, Austria. URL https://www.R-project.org/.
Ripley, B., 2014. Package “tree”. Retrieved from http://cran.rproject.org/web/packages/tree/index.html

RStudio Team, 2018. RStudio: Integrated Development for R. RStudio, Inc., Boston, MA URL http://www.rstudio.com/.

Schmidt, D., Chen, W., Matheson, M., Ostrouchov, G., 2017. "kazaam: Tools for Tall Distributed Matrices.” R

package version $0.1-0, \quad<$ URL: https://cran.rproject.org/package=kazaam $>$.

Sorg, L., Medina, N., Feldmeyer, D., Sanchez, A., Vojinovic, Z., Birkmann, J., Marchese, A., 2018. Capturing the multifaceted phenomena of socioeconomic vulnerability. In: Nat Hazards 92 (1), S. 257-282. DOI: 10.1007/s11069-018-3207-1.

Taiyun, W., Viliam, S., 2017. R package "corrplot": Visualization of a Correlation Matrix (Version 0.84). Available from https://github.com/taiyun/corrplot

Welle, T. \& Birkmann, J., 2015a. The WorldRiskIndex 2015. In WorldRiskReport 2015,pp. 41-49. Berlin: Alliance Development Works.

Welle, T. \& Birkmann, J., 2015b. The World Risk Index - An Approach to Assess Risk and Vulnerability on a Global Scale. Journal of Extreme Events, 02(01), 34 pages. doi.org/10.1142/s2345737615500037

Wickham, H., Ooms, J., Müller, K., 2018. RPostgres: 'Rcpp' Interface to 'PostgreSQL'. R package version 1.1.1. https://CRAN.R-project.org/package=RPostgres 